\title{
GASTO PÚBLICO EN INVERSIONES Y REDUCCIÓN DE LA POBREZA REGIONAL EN EL PERÚ, PERÍODO 2009-2018
}

\author{
PUBLIC EXPENDITURE ON INVESTMENTS AND REDUCTION OF REGIONAL \\ POVERTY IN PERU, PERIOD 2009-2018
}

Alipio Orco Díaz

Universidad Nacional Micaela Bastidas de Apurímac Abancay, Perú

ORCID: https://orcid.org/0000-0003-1307-0422 Correo electrónico: aorco@unamba.edu.pe

\section{RESUMEN}

Objetivo: Explicar la incidencia del gasto público en inversiones ejecutadas por funciones o sectores públicos en la reducción de la pobreza regional del Perú durante el periodo 2009-2018; y proponer un modelo econométrico que explique la variabilidad del nivel de pobreza en función a los cambios en el gasto público. Método: La investigación es de tipo cuantitativo, no experimental, longitudinal de panel, de alcance descriptivo y explicativo. La muestra está conformada por 240 observaciones del nivel de pobreza registrados en las 24 regiones del Perú, sin tomar en cuenta la provincia constitucional del Callao, durante el periodo comprendido del 2009 al 2018. Resultados: Los resultados de la investigación muestran una relación inversa de las variables, dado que el gasto público en inversiones reduce el nivel de pobreza regional; explicando en un $43,23 \%$ la variabilidad del nivel de pobreza regional en el Perú, durante el periodo 2009-2018. Asimismo, de un total de doce funciones que componen el gasto público, siete de ellas: agropecuario, comunicaciones, ambiente, saneamiento, vivienda, salud y educación, tienen coeficientes con signos negativos, que corresponden a lo teóricamente esperado. Conclusiones: Se concluye que el modelo econométrico estimado en conjunto explica en un $43,23 \%$ la variabilidad del nivel de la pobreza regional en el Perú y que las inversiones de las funciones: ambiental, saneamiento y educación tuvieron una incidencia significativa en la reducción de la pobreza en el Perú, periodo 2009-2018.

Palabras clave: Presupuesto; gasto público; pobreza; reducción; modelo; variabilidad.

\begin{abstract}
Objective: To explain the incidence of public spending on investments executed by public functions or public sectors in the reduction of regional poverty in Peru during the period 2009-2018; additionally, to propose an econometric model that explains the variability in poverty level based on changes in public spending. Method: The research is quantitative, non-experimental, of longitudinal panel, descriptive and explanatory. The sample consists of 240 observations in poverty level recorded in the 24 regions of Peru, without taking into account the constitutional province of Callao, during the period from 2009 to 2018. Results: The results of the investigation show an inverse relationship of the variables, given that public spending on investments reduces the level of regional poverty; explaining it in $43.23 \%$ the variability in regional poverty level in Peru, during the period 2009-2018. Also, of a total of twelve functions that make up public spending, seven of them are agriculture, communications, environment, sanitation, housing, health and education that present coefficients with negative signs, which correspond to what was theoretically expected. Conclusions: It is concluded that the estimated econometric model as a whole explains $43.23 \%$ of the variability in regional poverty level in Peru and that the investments on the functions: environmental, sanitation and education had a significant impact on the reduction of poverty in Peru, during the period 2009-2018.
\end{abstract}

Keywords: Budget; public spending; poverty; reduction; model; variability. 


\section{INTRODUCCIÓN}

El Perú ha logrado avances significativos en el crecimiento económico y en la prosperidad compartida en los últimos 15 años. El crecimiento económico estuvo acompañado por un incremento sostenido del gasto público en general y, en este contexto, se ha logrado una importante reducción en los niveles de pobreza. Desde 2005, según el Instituto Nacional de Estadística e Informática (INEI) la pobreza total se redujo de $55,6 \%$ a $20,5 \%$ en el 2018 .

Estos logros económicos y sociales fueron posibles en un contexto internacional, caracterizado por el incremento del precio de los minerales, componente principal de las exportaciones del Perú. En el periodo comprendido entre los años 2006 y 2015, una fortaleza de la economía peruana ha sido su estabilidad macroeconómica. El éxito económico, en este periodo, se expresa en el crecimiento del Producto Bruto Interno (PBI), en un promedio anual del 6\%; al pretender explicar este importante logro, podemos identificar un factor coyuntural y una acertada política económica. Como factor coyuntural nos referimos al incremento de los precios de exportación de los minerales en el mercado internacional. El otro aspecto de carácter endógeno fue la acertada política económica, reflejada en un adecuado control de la inflación, en un incremento de las recaudaciones fiscales, en un acertado manejo de la deuda pública, en una financiación de capitales a largo plazo y en un buen manejo del tipo de cambio, complementado con reformas institucionales (Banco Mundial, 2017).

La coyuntura económica favorable y la acertada política económica contribuyeron de manera significativa para que el Perú ejecute los más altos niveles de inversión pública, en comparación con sus pares latinoamericanos y con los países de la Organización de Cooperación y Desarrollo Económico (OCDE). La tendencia histórica en promedio, hasta el año 2007, fue del 3\% del PBI. Es a partir del año 2008, como parte de la política anticíclica, que el gasto público en inversiones se incrementó y se mantiene en promedio del 5,8\% del PBI. En términos absolutos, el gasto público en inversiones, en el periodo comprendido entre el año 2000 y 2007, fue de S/ 30756 120; este monto se multiplicó por 5,6 veces para el periodo comprendido entre 2008 y 2018.

La pobreza es un problema social de carácter eminentemente subjetivo, relativo y cambiante, por cuya razón adquiere diversas definiciones; pero, de manera general, se entiende como una condición en la cual una persona tiene un nivel de bienestar inferior, al mínimo socialmente aceptado. Para efectos de este trabajo de investigación, se utiliza el concepto de pobreza monetaria.
Según el Instituto Nacional de Estadística e Informática INEI (2019), se consideran como pobres monetarios:

A las personas que residen en hogares cuyo gasto per cápita es insuficiente para adquirir una canasta básica de alimentos y no alimentos (vivienda, vestido, educación, salud, transporte, etc.). Son pobres extremos aquellas personas que integran hogares cuyos gastos per cápita están por debajo del costo de la canasta básica de alimentos. (p. 39)

Para determinar la incidencia de la pobreza, el INEI utiliza el método de la línea de pobreza, que viene a ser un valor monetario con el cual se compara el gasto per cápita mensual de un hogar, y se considera pobre a aquel hogar cuyo gasto per cápita sea inferior a la línea de pobreza, y pobre extremo a aquel hogar cuyo gasto per cápita sea inferior a la línea de pobreza extrema.

En el periodo objeto de estudio (2009-2018), la incidencia de la pobreza tuvo un comportamiento decreciente. Inicialmente entre los años 2000 y 2004, en promedio el nivel de pobreza tiende a crecer y toca su punto máximo en el año 2004, con el 58,7\% de la población peruana en situación de pobreza. Es a partir del año 2005 que los niveles de pobreza en el Perú disminuyen de manera sostenida, reduciéndose para el año 2018 al 20,5\% de la población del país; en trece años la pobreza bajó 38,2 puntos porcentuales. No obstante, la pobreza rural sigue siendo alta con el $42,1 \%$ de pobreza, siendo tres veces más que en el área urbana $(14,4 \%)$.

Los diversos trabajos de investigación muestran que existen conexiones directas entre el gasto público en infraestructura y la reducción del nivel de pobreza. Uno de los primeros intentos sistemáticos de vincular la ejecución de proyectos de infraestructura con la reducción de la pobreza fue desarrollado por John Maynard Keynes en 1936. En su obra Teoría general del empleo, el interés y el dinero, Keynes argumenta que, en una economía caracterizada por la depresión y fallas del mercado, es necesaria la intervención del Estado a través del gasto público para reactivar la economía y elevar el nivel de empleo. Esto implica que una alta inversión pública en infraestructura aumenta el ingreso nacional, el empleo y el bienestar de las personas.

Para Wilhelm y Fiestas (2005), la política económica puede focalizar el gasto público hacia el crecimiento económico o puede enfocarse en reducir los niveles de pobreza. En el primer caso, al privilegiar el crecimiento económico, se estaría incrementando la demanda de mano de obra, oportunidad para incorporar a las personas pobres en las distintas actividades económicas, quienes incrementarían sus ingresos económicos con la posibilidad de salir de la pobreza. Si el enfoque es orientar el gasto público a reducir 
los niveles de pobreza, el gasto debería destinarse a la ejecución de proyectos de infraestructura productiva (transporte, electricidad o riego), debido a que la falta de estos bienes de capital podrían ser las principales causas de la condición de pobreza. Asimismo, se considera importante la participación del sector privado, a través de las distintas modalidades de inversión, complementando la inversión en infraestructura y servicios públicos.

Ogun (2010) analiza el impacto del desarrollo de infraestructura en la reducción de la pobreza en Nigeria. La investigación se enfoca en evaluar el efecto de la infraestructura física y social en los indicadores de pobreza. Para tal efecto hace uso de información estadística proporcionada por el Banco Central de Nigeria (2005) y la Oficina Nacional de Estadísticas de Nigeria, para el periodo 1970-2005, referidos al consumo per cápita real, como indicador de pobreza y la infraestructura agregada se aproxima por el gasto en infraestructura física representada por los gastos de capital real en los servicios económicos como: transporte, comunicación y carreteras; y la infraestructura social representada por los gastos de capital real en servicios sociales y comunitarios. Los resultados obtenidos sostienen que existe relación inversa entre la inversión en infraestructura y pobreza. El estudio también explica que la inversión en infraestructura social ejerce un mayor efecto en la reducción de la pobreza en relación con la inversión en infraestructura física. Concluye que la inversión masiva en infraestructura social en las ciudades reduciría drásticamente la pobreza en las áreas urbanas.

Chotia y Rao (2017) desarrollan un trabajo de investigación buscando evidencia empírica para responder a la pregunta de política sobre si el desarrollo de infraestructura puede contribuir en la reducción pobreza en Brasil, Rusia, India, China y Sudáfrica en el período 1991-2014. Los resultados obtenidos confirman que existe relación entre las variables consideradas y sugieren que existe evidencia de causalidad a corto plazo que va desde el crecimiento económico y el desarrollo de infraestructura hasta la reducción de la pobreza; además, afirman que el desarrollo de la infraestructura ayuda en reducir la pobreza.

Ali y Pernia (2003) sostienen que la inversión en infraestructura vial, eléctrica, de riego y, en general, infraestructura social y productiva, constituyen medios que permiten mejorar los ingresos de los habitantes de las zonas de influencia de dichos proyectos, al expandir sus fronteras productivas, facilitar acceso a mercados, aumentar productividad y generar ingresos por mano de obra, lo cual contribuye a reducir los niveles de pobreza.

En el Perú, se han realizado pocos trabajos de investigación relacionados con la medición de los impactos de la inversión en infraestructura básica y la reducción de los niveles de pobreza. La idea central es que las obras de infraestructura son importantes para el desarrollo económico y social; a través de ellas, un país puede incrementar su capacidad instalada necesaria para el desarrollo de sus actividades productivas. Así, tenemos que la construcción de mejores carreteras podría influir en la disminución de los costos logísticos y de transporte, la construcción de puertos también puede contribuir positivamente facilitando más las exportaciones; con mejores aeropuertos, el flujo de turistas al país podría ser mayor; con más centrales eléctricas, se podría mejorar la prestación del servicio eléctrico; y, en general, con más obras de infraestructura social y productiva, el país podrá mejorar de manera importante la prestación de los servicios públicos, impactando positivamente en el bienestar de la población (Albújar, 2016).

Las inversiones en infraestructura vial y de telecomunicaciones han contribuido en el incremento de los ingresos rurales, así como la productividad, a través, de la reducción del grado de aislamiento de la población y el incremento de la conexión. En el caso de la infraestructura vial, se afirma que ha logrado mejorar la transitabilidad, lo que reduce tiempos y costos, e incrementa el dinamismo de los mercados locales y regionales. Por su parte, la infraestructura de telecomunicaciones permitió la masificación del teléfono celular e internet, y logró que la proporción de hogares con teléfono celular en las áreas rurales pase de $2 \%$ en el 2005 a más de 50\% en el 2012 (Webb, 2013).

Fort y Paredes (2014) desarrollan la investigación Impacto de la inversión pública rural en el desarrollo de las regiones y niveles de bienestar de la población (2002-2012); el objetivo fue analizar los efectos de la inversión pública sobre la pobreza rural para el caso peruano a partir de información departamental (2004-2012). Los resultados de la investigación confirman que las inversiones en infraestructura de riego, caminos, telecomunicaciones y programas de apoyo al productor tienen un efecto significativo en la reducción de la pobreza. Asimismo, sostienen que los canales de impacto son a través de la mejora del capital humano, mejores niveles de conectividad, por tanto, el acceso a mercados más relevantes. Finalmente, resaltan la necesidad de aprovechar las complementariedades de las inversiones antes mencionadas para reducir la pobreza, así como mejorar la productividad agropecuaria.

En general, los diversos trabajos desarrollados en el Perú coinciden en sostener que las inversiones en infraestructura rural, como en riego, caminos, telecomunicaciones, apoyo al productor, electrificación, entre otros, tienen efectos significativos en la reducción del nivel de pobreza rural y sugieren que toda política pública antipobre debe considerar la necesidad de cerrar las brechas de infraestructura física en los diversos sectores económicos, procurando generar 
complementariedad de los diferentes proyectos de inversión rural, a fin de lograr un mayor efecto en la reducción de la pobreza.

En el caso del gasto público en programas sociales, sus presupuestos se incrementaron de manera sostenida entre 2009 y 2018. Son muy pocos los estudios sobre los impactos de los programas sociales en los niveles de pobreza en el Perú y los que existen solo corresponden al periodo comprendido entre el 2000 al 2010. Revisando estas investigaciones, observamos que las conclusiones de un primer grupo de investigadores resultan poco auspiciosas. Se evidencia un limitado impacto de los programas Vaso de Leche, Comedores Populares, Desayuno Escolar; no obstante, en el caso del Seguro Integral de Salud, esta conclusión es menos evidente por la falta de estudios centrados en el tema. Entre las diferentes causas a las que se atribuyen el limitado impacto de los programas sociales sobre los niveles de pobreza en el Perú, se mencionan: una deficiente gestión, alta infiltración, baja cobertura, politización del gasto, incremento de planillas de personal y gastos operativos. Se citan como ejemplos, entre los años 2002 y 2004, la subcobertura del programa Vaso de Leche estaría alrededor de 69\%, Comedores Populares 98\% y Desayunos Escolares 59\%; en cuanto a infiltración el Programa del Vaso de Leche se ubicaría en torno a $26 \%$ Comedores Populares 36\%, Desayunos escolares $46 \%$ y en el caso del Seguro Integral de Salud se estima una subcobertura de entre $50 \%$ y $70 \%$ y una infiltración de $25 \%$ a $30 \%$. Un segundo grupo de estudios afirman que los programas sociales sí estarían llegando a los pobres, pero no en las cantidades y dimensiones necesarias, con altos niveles de infiltración durante su gestión (Monge, Vásquez, y Winkelried, 2009).

Resumiendo, las conclusiones de los diferentes trabajos de investigación, observamos que no existe uniformidad en los resultados y, en algunos casos, son contrarios a lo esperado teóricamente. En este contexto, el objetivo de la investigación es explicar la incidencia del gasto público en inversiones ejecutadas por funciones o sectores públicos en la reducción de la pobreza regional del Perú durante el periodo 2009-2018 con el propósito de contribuir al debate académico, en la perspectiva de un gasto público más eficiente, asumiendo que es posible la reasignación presupuestal entre los diversos sectores, así como al interior de cada sector se pueden alcanzar mejoras de eficiencia con los recursos disponibles. Asimismo, en correspondencia al desarrollo teórico precedente, la hipótesis que guía esta investigación queda formulada del siguiente modo: El gasto público en inversiones, ejecutado por los diferentes sectores públicos, tiene una relación directa e inversa con el nivel de pobreza en el Perú, periodo 2009-2018.

\section{MATERIALES Y MÉTODOS}

La investigación fue de tipo cuantitativo, no experimental, longitudinal de panel, de alcance descriptivo y explicativo. Descriptivo, en tanto que se establecen los patrones del nivel de pobreza regional mediante estadísticos descriptivos y gráficos. Explicativo, en razón de que se busca conocer la relación del gasto en inversiones, según sectores públicos, sobre el nivel de la pobreza regional en el Perú en el periodo 2009-2018.

La muestra estuvo conformada por 240 observaciones del nivel de pobreza registrados en las 24 regiones del Perú, sin tomar en cuenta la provincia constitucional del Callao, durante el periodo comprendido del 2009 al 2018. El programa utilizado fue el estadístico STATA versión 14, debido a su versatilidad, amplias herramientas estadísticas y la diversidad de comandos para el análisis de estructura de datos de panel.

A continuación, se presenta el modelo econométrico planteado, para datos de panel:

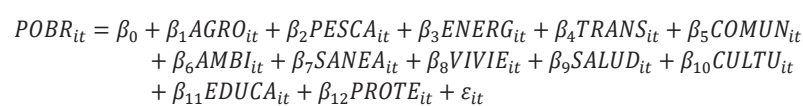

Dónde:

$\beta_{0}$ es la constante;

$\beta_{1}, \beta_{2}, \beta_{n}$ son estimadores;

POBR es el nivel de pobreza;

AGRO es el gasto público ejecutado por sector agropecuario; PESCA es el gasto público ejecutado por sector pesca; ENERG es el gasto público ejecutado por sector energía;

TRANS es el gasto público ejecutado por sector transportes; COMUN es el gasto público ejecutado por sector comunicaciones;

AMBI es el gasto público ejecutado por sector ambiente;

SANEA es el gasto público ejecutado por sector saneamiento;

VIVIE es el gasto público ejecutado por sector vivienda y desarrollo urbano;

SALUD es el gasto público ejecutado por sector salud;

CULTU es el gasto público ejecutado por sector cultura y deporte; 
EDUCA es el gasto público ejecutado por sector educación;

PROTE es el gasto público ejecutado por sector protección social.

Según este modelo econométrico, el nivel de pobreza depende del gasto público en inversiones de los diferentes sectores o funciones. Asimismo, el subíndice "i” hace referencia a las 24 regiones $\mathrm{y}$ " $\mathrm{t}$ " a los 10 años del periodo analizado.

\section{RESULTADOS}

A continuación, se muestran los resultados de la investigación.

En la tabla 1 se observa que el menor gasto público se dio en el año 2016 con 156 millones de soles y correspondió a la Región Tumbes; el mayor gasto público se registró durante el año 2018, con un gasto de 5920 millones de soles, ejecutado por la Región Lima. A nivel nacional el mayor gasto público anual se registró durante el 2018 con un monto de 28800 millones de soles.

Tabla 1

Máximos y mínimos del gasto público total por regiones, 2009-2018 (millones de soles)

\begin{tabular}{ccccc}
\hline Años & Mínimo & Promedio & Máximo & Total \\
\hline 2009 & 253 & 676 & 2420 & 16200 \\
2010 & 253 & 829 & 3460 & 19900 \\
2011 & 225 & 773 & 2980 & 18500 \\
2012 & 333 & 911 & 3430 & 21900 \\
2013 & 269 & 1030 & 3270 & 24700 \\
2014 & 290 & 1110 & 4640 & 26600 \\
2015 & 189 & 1010 & 4690 & 24200 \\
2016 & 156 & 956 & 4020 & 22900 \\
2017 & 193 & 1050 & 4150 & 25100 \\
2018 & 258 & 1200 & 5920 & 28800 \\
\hline
\end{tabular}

Fuente: Elaboración propia con información de transparencia económica - MEF

En la tabla 2, se muestra que el menor nivel de pobreza se dio en el año 2012, con un valor de 2,36\%, y se registró en la Región de Madre de Dios; el mayor nivel de pobreza fue de $73,87 \%$, correspondiente al año 2009 , registrado en la Región Apurímac. El valor de la mediana del nivel de pobreza, varía entre un máximo de $37,54 \%$ y un mínimo de 22,66\%, registrados en los años 2009 y 2016 respectivamente; mientras que el promedio máximo del valor del nivel de pobreza, fue de 39,46\%, registrado en el año 2009 y el promedio mínimo del valor del nivel de pobreza, fue de 23,67\%, registrado en el año 2018.
Tabla 2

Máximos y mínimos del nivel de pobreza regional, 20092018 (Var\%)

\begin{tabular}{ccccc}
\hline Años & Mínimo & Promedio & Mediana & Máximo \\
\hline 2009 & $6,13 \%$ & $39,46 \%$ & $37,54 \%$ & $73,87 \%$ \\
2010 & $5,01 \%$ & $35,05 \%$ & $37,51 \%$ & $62,52 \%$ \\
2011 & $4,15 \%$ & $30,88 \%$ & $28,51 \%$ & $54,92 \%$ \\
2012 & $2,36 \%$ & $28,93 \%$ & $26,44 \%$ & $53,31 \%$ \\
2013 & $4,53 \%$ & $27,51 \%$ & $26,05 \%$ & $50,54 \%$ \\
2014 & $4,09 \%$ & $26,59 \%$ & $25,37 \%$ & $50,27 \%$ \\
2015 & $4,96 \%$ & $25,07 \%$ & $26,90 \%$ & $48,19 \%$ \\
2016 & $3,03 \%$ & $23,96 \%$ & $22,66 \%$ & $47,36 \%$ \\
2017 & $3,47 \%$ & $24,00 \%$ & $24,64 \%$ & $47,53 \%$ \\
2018 & $3,12 \%$ & $23,67 \%$ & $24,98 \%$ & $46,66 \%$ \\
\hline
\end{tabular}

Fuente: Elaboración propia con información de SIRTOD - INEI

Del análisis del comportamiento histórico de estas dos variables, se colige que existe una relación inversa entre gasto público ejecutado y nivel de pobreza en el Perú para el periodo 2009-2018. La pobreza en las regiones ha venido disminuyendo cada año a partir del 2009 al 2018, cuyos valores fueron $39,46 \%$ y $23,67 \%$, respectivamente. El gasto público ejecutado en las regiones fue creciente a partir del año 2009 hasta el año 2018, cuyos gastos en inversiones fueron de S/ 16200 y S/ 28800 millones de soles, respectivamente (Figura 1).

A continuación, en la tabla 3 se presenta el Modelo de Datos de Panel de Efectos Fijos y Aleatorios estimados. Se escogió el modelo de efectos aleatorios por cuestiones de ajuste estadístico y mayor nivel inferencial, en razón de que su probabilidad Chi-cuadrada (0.0000) es menor que de los efectos fijos, al nivel de significación de 5\%, conforme lo establece el Test de Hausman.

Respecto al modelo de efectos aleatorios se concluye lo siguiente:

- Según el R cuadrado, en conjunto, las variables independientes utilizadas explican en un $43,23 \%$ la variabilidad del nivel de la pobreza regional.

- Se cumple la significancia individual para las variables independientes ambiente, saneamiento, educación, protección social y la constante.

- Se cumple la significancia conjunta del modelo, la probabilidad Chi-cuadrada (0.0000) es menor al nivel de significación de 5\%. En conjunto, las variables independientes sí explican significativamente el nivel de pobreza regional. 


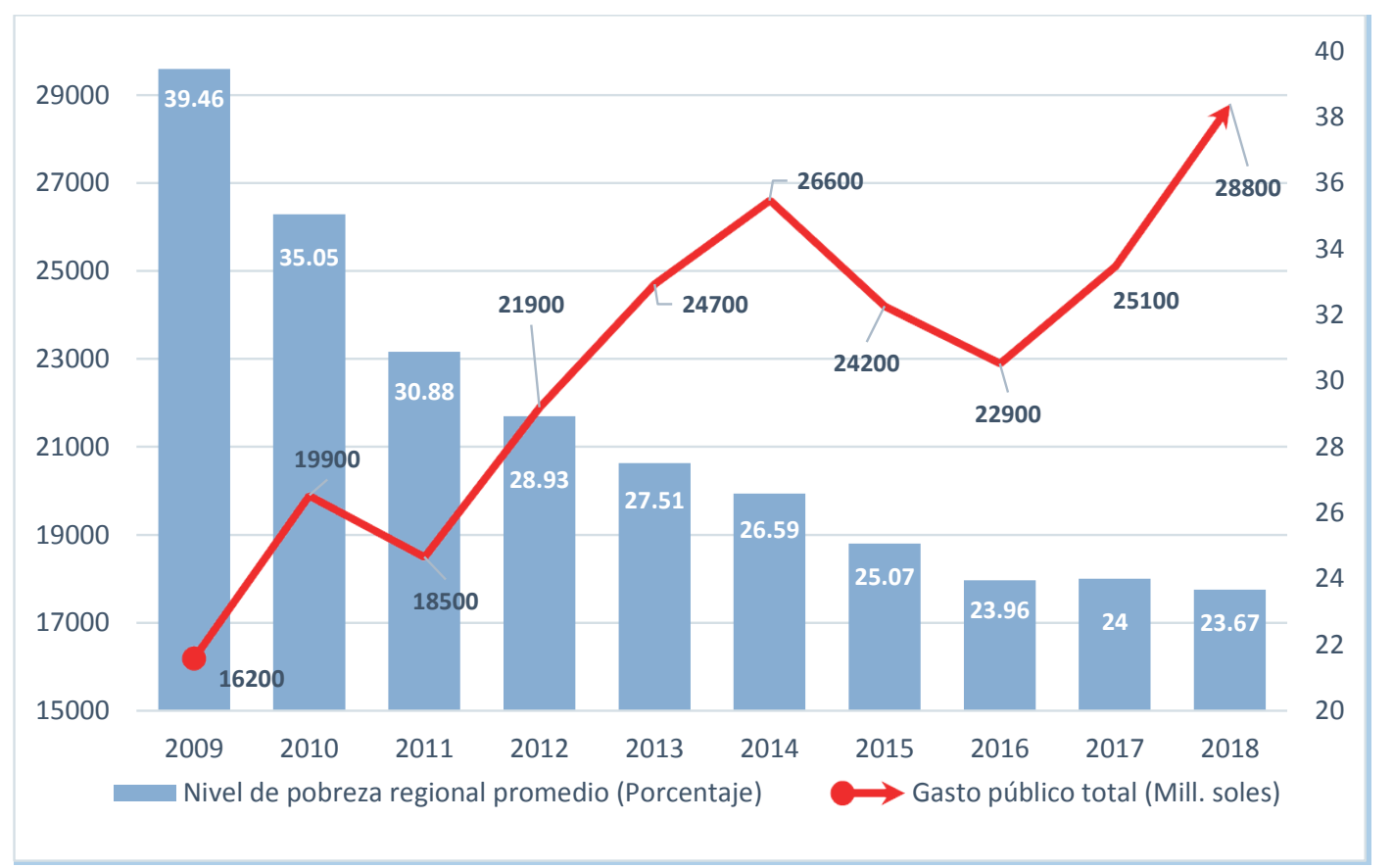

Figura 1. Gasto público y nivel de pobreza regional en el periodo 2009-2018. Fuente: Elaboración propia con información del INEI

Tabla 3

Modelo de datos de panel de efectos fijos y aleatorios

\begin{tabular}{|c|c|c|c|c|}
\hline & \multicolumn{2}{|c|}{ Modelo de efectos fijos } & \multicolumn{2}{|c|}{ Modelo de efectos aleatorios } \\
\hline GRO & $-7,40 \mathrm{E}-09$ & & $-6,52 \mathrm{E}-09$ & \\
\hline PESCA & 8,98E-08 & & $6,87 \mathrm{E}-08$ & \\
\hline ENERG & 8,64E-09 & & $3,71 \mathrm{E}-08$ & \\
\hline TRANS & $9,47 \mathrm{E}-10$ & & $7,87 \mathrm{E}-10$ & \\
\hline COMUN & $-1,19 \mathrm{E}-08$ & & $-8,03 E-09$ & \\
\hline AMBI & $-1,42 \mathrm{E}-07$ & $* * *$ & $-1,44 \mathrm{E}-07$ & $* * *$ \\
\hline SANEA & $-2,16 \mathrm{E}-08$ & $* * *$ & $-1,63 E-08$ & *** \\
\hline VIVIE & $-2,12 \mathrm{E}-08$ & * & $-2,33 E-08$ & * \\
\hline SALUD & $-4,97 \mathrm{E}-09$ & & $-6,96 \mathrm{E}-09$ & \\
\hline CULTU & 1,74E-08 & * & 1,77E-08 & * \\
\hline EDUCA & $-4,02 \mathrm{E}-08$ & *** & $-3,41 \mathrm{E}-08$ & *** \\
\hline PROTE & 3,77E-07 & $* \star \star$ & 3,68E-07 & $* \star *$ \\
\hline Constante & 36,56412 & $* * *$ & 34,66778 & $* * *$ \\
\hline R Square & $43,87 \%$ & & $43,23 \%$ & \\
\hline Wald $\mathrm{Chi}^{2}(12)$ & & & 127,53 & \\
\hline Prob. Chi & & & 0,0000 & \\
\hline$F(12,204)$ & 13,29 & & & \\
\hline Prob. F & 0,0000 & & & \\
\hline \multicolumn{5}{|c|}{ Test de Hausman } \\
\hline $\mathrm{Chi}^{2}(12)$ & 42,12 & & & \\
\hline Prob. Chi ${ }^{2}$ & 0,0000 & & & \\
\hline
\end{tabular}

Nota: ${ }^{* * *}$ menor a $1 \%,{ }^{* *}$ menor a $5 \% \mathrm{y}$ * menor a $10 \%$ del nivel de significancia.

Fuente: Elaboración propia. 
- Con los coeficientes del modelo de efectos aleatorios se desarrolló el siguiente modelo econométrico, que explica la incidencia del gasto público ejecutado por funciones en el nivel de pobreza regional del Perú, periodo 2009-2018.

$$
\begin{aligned}
& P_{O B R_{i t}}=36,56412-7,40 \mathrm{E}-09 * A G R O_{i t}+8,98 \mathrm{E}-08 \\
& * P_{E S C A}+8,64 \mathrm{E}-09 * E N E R G_{i t}+9,47 \mathrm{E}-10 \\
& * \text { TRANS }_{i t}-1,19 \mathrm{E}-08 * \text { COMUN }_{i t}-1,42 \mathrm{E}-07 \\
& \text { * AMBI } I_{i t}-2,16 \mathrm{E}-08 * S A N E A_{i t}-2,12 \mathrm{E}-08 \\
& * V I V I E_{i t}-4,97 \mathrm{E}-09 * S A L U D_{i t}+1,74 \mathrm{E}-08 \\
& \text { *CULTU } U_{i t}-4,02 \mathrm{E}-08 * E D U C A_{i t}+3,77 \mathrm{E} \\
& -07 * \text { PROTE }_{i t}
\end{aligned}
$$

El modelo econométrico estimado es una representación simplificada de la relación entre gasto público ejecutado y nivel de pobreza para el periodo 2009-2018, cuyo uso permite hacer predicciones del valor futuro del nivel de pobre$\mathrm{za}$, como consecuencia de las variaciones del gasto público. La aplicación de este modelo econométrico en los procesos de programación multianual de inversiones (PMI), en el sector público peruano, facilitaría la evaluación del impacto de los proyectos de inversión en los niveles de pobreza, incorporando en estos procesos criterios técnicos en lugar de procedimientos eminentemente políticos.

Según los estimadores significativos del modelo econométrico de efectos aleatorios se formula las siguientes inferencias:

- Si el gasto público del sector ambiente se incrementara en un millón de soles, entonces el nivel de pobreza regional disminuiría aproximadamente en $0,142 \%$.

- Si el gasto público del sector saneamiento aumentara en un millón de soles, entonces el nivel de pobreza regional disminuiría aproximadamente en $0,0216 \%$.

- Si el gasto público del sector educación aumentara en un millón de soles, entonces el nivel de pobreza regional disminuiría en 0,0402\%.

- Si no hubiera inversión pública, es decir que las variables independientes fueran igual a cero, entonces el nivel de pobreza regional se mantendría constante en $36,56412 \%$.

\section{DISCUSIÓN}

Como el objetivo del artículo es explicar la incidencia del gasto público en inversiones ejecutadas por funciones o sectores públicos en la reducción de la pobreza regional del Perú durante el periodo 2009-2018; los resultados obtenidos en la tabla 3 , muestran una relación inversa de las variables, dado que el gasto público en inversiones reduce el nivel de pobreza regional; explica en conjunto en un $43,23 \%$ la variabilidad del nivel de pobreza regional en el Perú, durante el periodo 2009-2018. Asimismo, de un total de doce funciones o sectores que componen el gasto público, siete funciones o sectores: agropecuario, comunicaciones, ambiente, saneamiento, vivienda, salud y educación; tienen coeficientes con signos negativos, que corresponden a lo teóricamente esperado. Estos resultados son consistentes con los obtenidos por Fort y Paredes (2014), cuya principal conclusión es que encuentran incrementos en la productividad agrícola y efectos significativos sobre la pobreza rural, como impactos de la inversión pública rural en proyectos de riego, caminos y telecomunicaciones; de esto se desprende que existe una relación inversa entre inversión y pobreza. Asimismo, los resultados de esta investigación, concuerdan con los de Ali y Pernia (2003), quienes sostienen que la inversión en infraestructura vial, eléctrica, de riego y, en general, en infraestructura social y productiva, constituyen medios que permiten mejorar los ingresos de los habitantes de las zonas de influencia de dichos proyectos, al expandir sus fronteras productivas, facilitar acceso a mercados, aumentar productividad y generar ingresos por mano de obra; $y$ de este modo, contribuyen a reducir los niveles de pobreza. El otro grupo de funciones o sectores constituidos por pesca, energía, transporte y cultura tienen coeficientes de signo positivo, contrarios a los esperados, dado que la teoría en general afirma que a más inversión corresponde un mayor crecimiento económico, por consiguiente, se debe reducir la pobreza. Sin embargo, a pesar de haberse dado en estos sectores, mayores niveles de inversión y sostenido crecimiento económico, los resultados obtenidos demuestran que no tienen incidencia en la reducción del nivel de pobreza. Tal situación se puede explicar en el sentido de que el gasto público en estos sectores no necesariamente está diseñado para reducir la pobreza, probablemente el interés político mayor sea el crecimiento económico, y que se requieren de políticas económicas redistributivas propobres más agresivas. La inversión en protección social, que comprende a todos los programas sociales de lucha contra la pobreza, muestra un coeficiente de signo positivo, el cual resulta también bastante controversial y discutible. Teóricamente se asume que los programas sociales reducen la pobreza, sin embargo, el resultado obtenido es contrario a la teoría. La explicación de estos resultados, conduce a suponer que podría obedecer a las elevadas tasas de infiltración (porcentaje de usuarios de programas que no son parte de la población objetivo) y a la subcobertura (el porcentaje de la población objetivo que no es atendida); razonamiento concordante con los resultados obtenidos por Monge et al. (2009). En síntesis, el gasto 
publico ejecutado en inversiones por los sectores o funciones, en el periodo 2009-2018, explican en un 43,23\% la variabilidad del nivel de pobreza en el Perú. Respecto a la incidencia de la inversión ejecutada por cada sector, se tiene que las funciones: agropecuario, comunicaciones, ambiente, saneamiento, vivienda, salud y educación reducen el nivel de pobreza. Sin embargo, cabe resaltar que los sectores: ambiente, saneamiento y educación contribuyen de manera significativa.

\section{REFERENCIAS}

Albújar, A. (2016). Medición del impacto en la economía de la inversión en infraestructura público-privada en países en vías de desarrollo. Tesis Doctoral. Universitat Ramon Llull, España. Recuperado de http://hdl.handle.net/10803/352465

Ali, I. y Pernia, E. (2003). Infrastructure and poverty reduction. What is the Connection? Asian Development Bank. ERD Policy Brief, 13. Recuperado de

Banco Mundial (2017). Perú. Revisión del gasto público. Recuperado de http://documentos.bancomundial. org/curated/es/554021521229272108/pdf/Peru-Revision-del-Gasto-Publico-2018-final.pdf

Chotia, V. y Rao, M. (2017). Investigating the interlinkages between infrastructure development, poverty and ruralurban income inequality: Evidence from BRICS nations. Studies in Economics and Finance, 34(4), 466-484, https://doi.org/10.1108/SEF-07-2016-0159

Fort, R. y Paredes, H. (2014). Impacto de la Inversión Pública Rural en el desarrollo de las regiones y bienestar de la población (2002-2012). Lima: GRADE. Recuperado de http://www.cies.org.pe/sites/default/files/investigaciones/informe_final_cies_2910.pdf
Instituto Nacional de Estadística e Informática. (2019). Informe Técnico: Evolución de la pobreza monetaria 2007-2018. Lima: INEI. Recuperado de https://www. inei.gob.pe/media/MenuRecursivo/publicaciones_ digitales/Est/Lib1646/libro.pdf

Keynes, J. (1970). Teoría general del empleo, el interés y el dinero. México: Fondo de Cultura Económica.

Monge, A. Vásquez, E. y Winkelried, D. (2009). ¿Es el gasto público en programas sociales regresivo en el Perú? Lima: Ministerio de Economía y Finanzas del Perú. Recuperado de https://www.mef.gob.pe/contenidos/pol_econ/ documentos/Gasto_en_programas_sociales.pdf

Ogun, T. (2010). Infrastructure and poverty reduction: Implications for urban development in Nigeria. Urban Forum, 21(3), 249-266. https://doi.org/10.1007/s12132010-9091-8

Webb, R. (2013). Conexión y Despegue Rural. Lima: Universidad San Martín de Porres. Recuperado de http://www.lampadia.com/assets/uploads_librosdigitales/2f207-cdr.pdf

Wilhelm, V. y Fiestas I. (2005). Exploring the Link between Public Spending and Poverty Reduction: Lessons from the 90s. Washington, D.C.: World Bank Institute Working Papers. Recuperado de http://documents. worldbank.org/curated/en/ 501641468324280601/pdf/358680WBI0rev01ng0the0Link01PUBLIC1.pdf 